\title{
LUPUS SHRINKING LUNG SYNDROME WITH GOOD RESPONSE WITH CORTICOSTEROID AND METHOTREXATE: A CASE REPORT
}

Filipe Mateus Oliveira LIma (Universidade Federal de Pernambuco, Recife, PE, Brasil), Bruno Rodrigo

Fontes (Universidade Federal de Pernambuco, Recife, PE, Brasil), Daniel Neri Matta (Universidade Federal de Pernambuco, Recife, PE, Brasil), Rafaela Silva Guimaraes Gonçalves (Universidade Federal de Pernambuco, Recife, PE, Brasil), Claudia Diniz Lopes Marques (Universidade Federal de Pernambuco, Recife, PE, Brasil), Nara Gualberto Cavalcanti (Universidade Federal de Pernambuco, Recife, PE, Brasil), Aline Ranzolin (Universidade Federal de Pernambuco, Recife, PE, Brasil), Sergio Cavalcanti (Universidade Federal de Pernambuco, Recife, PE, Brasil), Henrique Ataide Mariz (Universidade Federal de Pernambuco, Recife, PE, Brasil), Angela Luzia Branco Pinto Duarte (Universidade Federal de Pernambuco, Recife, PE, Brasil)

\section{BACKGROUND}

Shrinking lung syndrome (SLS) is a rare pleuropulmonary manifestation of systemic lupus erythematosus (SLE) characterized by diaphragmatic dysfunction and a restrictive pattern on pulmonary function testing with normal pulmonary parenchyma. The estimated prevalence ranges between 0.5 and $10 \%$. Although the cause of SLS remains controversial, one possible mechanism is diaphragm 's myositis or myopathy resulting in its elevation and decreased function. Factors associated with SLS include greater disease duration, seropositivity for anti-RNP antibodies and a history of pleuritis. Clinically, patients present with progressive dyspnea. Although there is low level of evidence supporting the use of immunosuppressive therapy for SLS, glucocorticoids and immunosuppressive therapy (cyclophosphamide, mycophenolate and azathioprine) may improve both symptoms and pulmonary function. Noninvasive positive pressure ventilation may also be beneficial We describe the case of a patient with SLE who manifested SLS soon after the diagnosis and presented a good response with the treatment performed

\section{CASE REPORT}

A 46-year-old woman, who was recently diagnosed with SLE, presenting a one-year history of mild polyarthralgia, malar rash, Raynaud's phenomenon and positive ANA. She, was hospitalized with pleuritic chest pain and progressive dyspnea without fever, cough or weight of loss. The symptoms became worse when supine position The patient reported good health until five months before, when a similar clinical presentation of pleuritic pain and dyspnea was developed. At the time, she took antibiotics and corticosteroids, evolving with pain relief but continued with dyspnea. Physical examination on admission revealed abdominal paradox, decreased breath sounds with crackles at the pulmonary bases. Laboratory was essentially normal, except for high sedimentation rate, positive anti-DNA and anti-RNP. Chest X-ray showed elevation of right hemidiaphragm, reduced lung fields, and opacification of right hemi thorax lung base. Additionally, chest computed tomography revealed a small right pleural effusion and basilar atelectasis on both lungs. Pulmonary function testing showed restrictive pulmonary defect. After treatment with $60 \mathrm{mg}$ prednisone, methotrexate and hydroxychloroquine, chest pain and breathlessness gradually improved, as well as the radiographic findings

\section{CONCLUSION}

: Although it is a rare manifestation of SLE, SLS should be considered as a differential diagnosis of dyspnea in these patients. In the present case, early treatment with steroids and immunosuppressive medication presented a good clinical response with total symptoms resolution 